\title{
OPEN Effectiveness of an exercise-based prehabilitation program for patients awaiting surgery for lumbar spinal stenosis: a randomized clinical trial
}

\author{
Andrée-Anne Marchand ${ }^{1 凶}$, Mariève Houle ${ }^{2}$, Julie 'Shaughnessy $^{1}$, \\ Claude-Édouard Châtillon ${ }^{3,4}$, Vincent Cantin ${ }^{5}$ \& Martin Descarreaux ${ }^{5}$
}

Lumbar spinal stenosis is the most common reason for spine surgery in older adults, but the effects of prehabilitation on perioperative outcomes among these patients have not been investigated. This study aims to evaluate the effectiveness of a preoperative exercise-based intervention program compared with usual care on the improvement of clinical status, physical capacities and postoperative recovery of patients awaiting surgery for lumbar spinal stenosis. Sixty-eight participants were randomised to receive either a 6-week supervised exercise-based prehabilitation program or hospital usual care. The outcomes included both clinical and physical measures. Data collection occurred at post-intervention, and 6 weeks, 3 - and 6 -months post-surgery. Significant but small improvements were found in favour of the experimental group at the post-intervention assessment for pain intensity, lumbar spinal stenosis-related disability, lumbar strength in flexion, low back extensor muscles endurance, total ambulation time, and sit to stand performance. A significant difference in favor of the intervention group was found starting at the 3-month postoperative follow-up for low back-related disability. No adverse events were reported. Exercise-based prehabilitation did not improve shortterm postoperative recovery in patients with lumbar spinal stenosis.

As populations worldwide continue to grow older, increasing demand is to be expected on health care systems, including surgery-related resources. The metabolic response that results from surgical procedures is characterized by changes in body composition, loss of muscle function and strength, decreased vasomotor control, and sympathetic hyperactivity ${ }^{1,2}$. Considering that the extent of this response impacts postoperative recovery and long-term outcomes, attempts to minimize it have become paramount for the perioperative teams ${ }^{3,4}$.

The aging process is naturally associated with some degree of physical deconditioning ${ }^{5,6}$, which compromises the physiologic reserve required to better withstand the stress of surgery ${ }^{7}$. Furthermore, loss of physiologic reserve is aggravated by sedentary behavior that in turn contributes to negative consequences on functional independence ${ }^{8}$. Physical exercise, including resistance and aerobic training, is part of the solution to attenuate physical decline in older individuals ${ }^{9,10}$. As such, interventions aimed at augmenting patients' physiological fitness prior to a surgical intervention to allow them to increase their metabolic reserve and retain a higher level of functional capacity over their entire perioperative trajectory, are known as prehabilitation ${ }^{8,11}$.

Most publications on prehabilitation pertain to life-threatening diseases and complex surgeries while the body of evidence in the context of elective spine surgery remains scarce ${ }^{12-14}$. In a recent systematic review, Janssen et al. reported on the effectiveness of prehabilitation in patients with degenerative disorders of the lumbar spine $^{15}$. Based on data from twelve interventions, the authors concluded that prehabilitation has no effect when compared to usual care in patients undergoing lumbar spinal surgery. However, only one of the included studies

${ }^{1}$ Department of Chiropractic, Université du Québec à Trois-Rivières, 3351 Boul. des Forges, Trois-Rivières, OC G9A $5 \mathrm{H7}$, Canada. ${ }^{2}$ Department of Anatomy, Université du Québec à Trois-Rivières, Trois-Rivières, Canada. ${ }^{3}$ Centre Intégré Universitaire de Santé et de Services Sociaux de la Mauricie-et-du-Centre-du-Québec, Trois-Rivières, Canada. ${ }^{4}$ Division of Neurosurgery, Faculty of Medicine, University of Montreal, Montréal, Canada. ${ }^{5}$ Department of Human Kinetics, Université du Québec à Trois-Rivières, Trois-Rivières, Canada. ${ }^{\circledR}$ email: andree-anne.marchand@ uqtr.ca 
(with unclear risk of bias) looked at exercise ${ }^{16}$, which was not enough to draw any conclusion regarding the effectiveness of exercise-based prehabilitation ${ }^{15}$.

Lumbar spinal stenosis (LSS) is one of the most frequent degenerative conditions in older-aged patients ${ }^{17}$ and represents the main reason for undergoing surgery in adults over the age of $65^{18}$. It is hallmarked by neurogenic claudication, causing high levels of disability, disrupting activities of daily leaving and leading to a more sedentary lifestyle ${ }^{19}$. With as little as $4 \%$ of patients meeting the Canadian recommendations for physical activity ${ }^{20}$ and considering that watchful-waiting is safe in this slowly progressing condition ${ }^{21}$, LSS may be best suited to study the effect of prehabilitation prior to spine surgery.

Therefore, the aim of the study was to assess the effectiveness of an active exercise-based prehabilitation programme compared to usual care in patients with LSS. It was hypothesized that patients in the intervention group would have greater preoperative functional capacities, which would lead to faster post-operative recovery, compared to the control group.

\section{Methods}

Study design. The study was a single-centre, parallel-group randomized controlled trial with an internal pilot component. We previously conducted a pilot study to test the intervention, the choice of outcome measures, and to gather preliminary data. Given that the intervention was not modified between the pilot and the main trial, the forty participants from the pilot study were included in the final analysis presented herein. The trial was conducted at the Université du Québec à Trois-Rivières (UQTR) research facility, Canada. Enrollment started in February 2015, with the last follow-up in February 2020. The trial protocol ${ }^{22}$ as well as the feasibility and pilot results ${ }^{23}$ have been published elsewhere. The study received ethical approval from the institutional review board of UQTR (CÉR-2014-008-00) and was registered in ClinicalTrials.gov (NCT02258672; October $7 \mathrm{th}, 2014)$. All methods were carried out in accordance with relevant guidelines and regulations. Informed written consent was obtained from each participant prior to data collection.

Participants. We included individuals $\geq 18$ years, diagnosed with degenerative LSS primarily of central origin (confirmed with matching clinical history and diagnostic imaging), awaiting surgery (minimally invasive or open approach) and able to provide written informed consent voluntarily. Exclusion criteria included presence of non-degenerative LSS, inflammatory arthritic conditions, vertebral instability requiring non-instrumental or instrumented fusion and altered cognitive capacities; individuals deemed ineligible by their treating neurosurgeon; and being unable to understand or express oneself in French. All patients were recruited at the Centre intégré universitaire de santé et de services sociaux de la Mauricie-et-du-Centre-du-Québec (Trois-Rivières' regional hospital (Quebec, Canada)) in collaboration with the neurosurgery team. Neurosurgeons were responsible for identifying eligible patients during outpatient clinical encounters. Patients meeting inclusion criteria and interested in the study were asked for consent to be later contacted by a member of the research team.

Interventions. All participants, regardless of group allocation, received the day prior to surgery, standardized written information on how to keep a good back posture when getting in or out of bed and when sitting down after the surgery. That is the usual care provided by the hospital staff for all patients undergoing back surgery.

Participants in the exercise group were offered individually supervised exercise sessions 3 times per week for 6 weeks, prior to their surgery. Training sessions took place at the Université $d u$ Québec à Trois-Rivières rehabilitation facility and were led by a certified kinesiologist. A typical training consisted of a 5-min warm-up (stationary cycling or walking on a treadmill based on participants' preference), followed by 25 min of exercises with concentric or isometric phases that aimed to improve muscle and structures involved in walking capacities. Exercise intensity level was individually tailored to the participants' capacity and progressively modified to provide increasing levels of difficulty. For a full description of the exercise intervention, see previous reports ${ }^{22,23}$. Adherence to the exercise program was documented in the kinesiologist logbook. For each exercise, recorded data include the number of sets, repetitions and levels of difficulty reached (1 being the lowest and 4 the highest level of difficulty), perceived effort and location, intensity, and character of discomfort if any. Participants in the control group were not discouraged from performing physical activity or exercise.

Outcomes. Sociodemographic data were collected via a structured interview and by self-reported questionnaires at baseline, with a trial researcher available to clarify questions if needed.

Treatment effect was assessed using both clinical patient-reported outcome measures and objective physical tests. Clinical patient-reported outcome measures were collected at UQTR's research facility at baseline, 6-week from baseline (post-intervention), and 6 weeks post-surgery, and by post at 3- and 6-month post-surgery. Physical outcome measures were collected at UQTR's research facility at baseline, after 6 weeks prehabilitation intervention, and 6 weeks post-surgery.

Primary outcome measures were current low back and leg pain intensity (11-point Numerical Rating Scale) ${ }^{24}$, and low back-related disability (Oswestry Disability Index $)^{25}$.

Secondary outcome measures included quality of life (EuroQol-5D) ${ }^{26}$, fear avoidance behavior (Tampa Scale of Kinesiophobia) $)^{27}$, level of anxiety and depression (Beck Disability Index) ${ }^{28}$, patient perception of treatment effect (7-point scale Patient Global Impression of Change ${ }^{29}$-measured at the post-intervention assessment only), lumbar extensor muscles endurance (modified Sorensen test) ${ }^{30}$, trunk flexor and extensor muscle strength (isometric contraction) $)^{31}$, knees extensor muscle strength (isometric contraction) ${ }^{32}$, active lumbar ranges of motion ${ }^{33}$, and walking abilities (time to first symptoms and total ambulation time). Lastly, perioperative data including blood loss, length of surgery, surgical technique used, intraoperative complications, and length of hospital stay 
were documented as potential explanatory factors of between group differences in recovery. The study protocol provides further information about the selected outcomes ${ }^{22}$.

Based on results from the pilot study ${ }^{23}$, physical tests better reflecting patients' activities of daily living were deemed necessary to capture functional capacities as oppose to physiological changes to exercise. As such, in the main trial, we included the $30 \mathrm{~s}$ sit-to-stand ${ }^{34}$ and the timed up and go ${ }^{35}$ tests that allowed for the measurement of progress regarding balance, sit to stand, and short-distance walking capacities. In addition, in response to the participants comment that the ODI did not completely capture their daily challenges we also added the French Swiss Spinal Stenosis questionnaire ${ }^{36}$ to measure LSS-related disability. These newly collected outcomes are available for the latest recruited participants only (15 and 13 from the intervention and control group respectively).

Sample size. The sample size calculation for the main trial was conducted using the pilot study's means and standard deviations for leg pain intensity ${ }^{23}$ (measured after the 6-week prehabilitation intervention), assuming a one-tailed test and considering a significance level of $\mathrm{p}=0.01$, a power of $90 \%$, and a $20 \%$ attrition rate. An estimated 36 patients per treatment arm were required to detect a significant between-group difference.

Randomization and blinding. Randomisation and minimization were performed after the baseline assessment using a computer random number generator, prepared by a research assistant not involved in the study process. Allocation concealment was ensured using sequentially numbered, opaque and sealed envelopes. The envelopes were opened in front of the participants by the main investigator after enrollment. Participants were not blinded to intervention allocation, but to prevent cross-contamination between groups content of exercise sessions was known only to those in the intervention group. Further details about randomisation, minimization and blinding are published elsewhere ${ }^{22,23}$.

Statistical methods. For between-group comparisons of demographic and perioperative data, the independent Student $t$ test for continuous variables and the chi-square test for categorical variables were used. Mixed model ANOVAs were used for group comparison over time and Bonferroni post hoc tests were conducted whenever necessary. Based on observations from the pilot study during which a significant effect of surgery was observed for primary clinical outcomes ${ }^{23}$, the analyses were first conducted using the baseline and postintervention data, and then the post-intervention and follow-ups data together. Whenever baseline variables did not follow normal distribution using the Shapiro-Wilk test, appropriate transformations were applied in order to conduct parametric statistics. Analyses of primary and secondary outcomes were conducted according to the intention-to-treat principle with participants analyzed according to randomly assigned treatment group irrespective of compliance. Missing data (mean number $=19.1 \%$ per table) were replaced using multiple imputation regression modeling methods and an aggregate of 1000 imputed data sets was used to conduct the analysis of variance. All analyses were conducted in SPSS Statistics version 25.0. (IBM, Armonk, NY). The level of significance was set to 0.05 .

\section{Results}

Recruitment. Between February 2015 and June 2019, a total of 98 eligible patients were contacted, of whom 68 agreed to participate and were randomly assigned to the intervention $(n=35)$ or control group $(n=33)$. Due to the long follow-up period and a much lower patient load during summer months for the neurosurgeons, it was decided to stop the recruitment prematurely, with $94 \%$ of the recruitment goal achieved. Figure 1 presents participants flow in the study along with reasons for non-participation and attrition.

Baseline data. There was no significant difference between the groups with respect to baseline characteristics except for age which was lower $(\mathrm{p}=0.01)$ in the intervention group. Table 1 presents the baseline characteristics for all participants.

Participants' adherence to intervention. A total of 14 participants completed all 18 training sessions as planned (40\% compliance) whereas 17 completed more than $50 \%$ of sessions (range 10-17), and 4 less than $50 \%$ (range 2-7). Considering that the intervention period was shortened for some participants due to the variable rate of surgical operation for elective surgeries, we can consider that a maximum of 569 sessions could be provided to participants yielding a compliance rate of $90.3 \%$ (288/569) with a mean of 14.7 sessions provided per participant. Assessment of physical activities performed outside of the study protocol at the post-intervention assessment was similar in both groups $(\mathrm{p}=0.39)$ with 8 and 10 participants reporting being active in the intervention and control group respectively. (Results based on 29 individuals in the intervention group and 26 in the control group). Types of physical activity included treadmill or outdoor walking, stationary or outdoor cycling, snowshoeing, fall risk prevention program, and performing the prehabilitation exercises on off days.

Participants' adherence to surgical plan. Out of the 68 enrolled participants, 4 did not undergo surgery as planned. All 3 individuals from the control group opted out because the risks associated with the surgery were perceived as too high given their advanced age or concomitant health issues. The one individual that opted out of surgery in the intervention group did so in accordance with the neurosurgeon's opinion that her functional status had improved beyond surgical candidacy. In total 64 participants underwent lumbar laminectomy/ laminotomy over the course of the study and none underwent a revision surgery within the 6-month follow-up. 


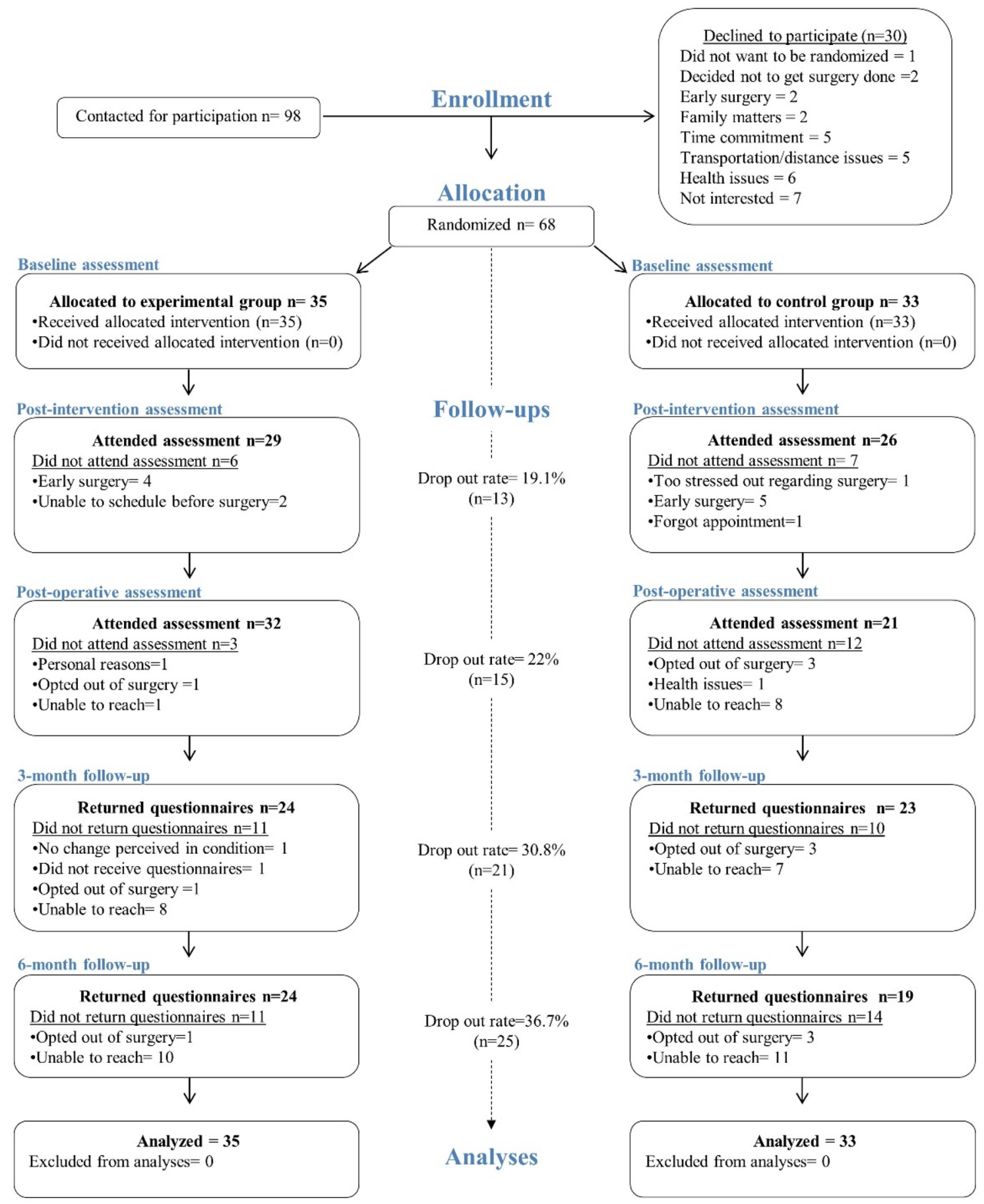

Figure 1. CONSORT flowchart of the randomized controlled trial.

Outcomes and estimation of intervention effect. Results of analyses conducted using preoperative data. Significant Group $\times$ Time interactions were found between the baseline and the preoperative assessments in favor of the intervention group for leg pain intensity $\left(\mathrm{F}_{1,66}=4.63, \mathrm{p}=0.03, \eta \mathrm{p}^{2}=0.07\right)$; LSS-related disability $\left(\mathrm{F}_{1,25}=4.48, \mathrm{p}=0.04, \eta \mathrm{p}^{2}=0.15\right)$; maximum lumbar strength in flexion $\left(\mathrm{F}_{1,66}=5.15, \mathrm{p}=0.02, \eta \mathrm{p}^{2}=0.07\right)$; low back extensor muscles endurance $\left(\mathrm{F}_{1,63}=6.67, \mathrm{p}=0.002, \eta \mathrm{p}^{2}=0.15\right)$; total ambulation time $\left(\mathrm{F}_{1,64}=5.63, \mathrm{p}=0.02\right.$, $\left.\eta \mathrm{p}^{2}=0.08\right)$; and sit to stand $\left(\mathrm{F}_{1,25}=7.02, \mathrm{p}=0.01, \eta \mathrm{p}^{2}=0.22\right)$. Figures 2,3 and 4 present between group comparisons of low back related disability, leg and back pain intensity and walking capacities respectively.

Results of analyses conducted using postoperative data. The only significant Group $\times$ Time interaction found in the postoperative period, was for low back-related disability in favor of the intervention group $\left(\mathrm{F}_{2,132}=6.20\right.$, $\left.\mathrm{p}=0.003, \eta \mathrm{p}^{2}=0.06\right)$ with the largest difference being at 6 months. Means, standard deviations and $95 \%$ con- 


\begin{tabular}{|c|c|c|c|}
\hline & $\begin{array}{l}\text { Intervention }(\mathrm{N}=35) \\
\text { Mean } \pm \text { SD }\end{array}$ & $\begin{array}{l}\text { Control }(\mathrm{N}=33) \\
\text { Mean } \pm \text { SD }\end{array}$ & $p$ \\
\hline \multicolumn{4}{|l|}{ Demographics } \\
\hline Age-years & $66.2 \pm 9.6$ & $71.6 \pm 7.6$ & $0.01^{*}$ \\
\hline Gender, female-n (\%) & $14(40)$ & $14(42)$ & 0.83 \\
\hline BMI & $29.0 \pm 5.2$ & $28.9 \pm 5.2$ & 0.90 \\
\hline Weight-kg & $81.3 \pm 16.5$ & $82.7 \pm 19.1$ & 0.74 \\
\hline Height-cm & $169.9 \pm 11.7$ & $166.2 \pm 10.0$ & 0.16 \\
\hline \multicolumn{4}{|l|}{ Minimisation criteria } \\
\hline Diabetes-n (\%) & $4(11)$ & $7(21)$ & 0.27 \\
\hline Positive EMG findings-n (\%) & $7(20)$ & $3(9)$ & 0.20 \\
\hline ODI score $\geq 41 \%-\mathrm{n}(\%)$ & $14(40)$ & $9(27)$ & 0.26 \\
\hline Smoker-n (\%) & $2(5)$ & $1(3)$ & 0.59 \\
\hline \multicolumn{3}{|l|}{ Employment situation-n (\%) } & 0.39 \\
\hline Currently working & $3(8.6)$ & $5(15.2)$ & \\
\hline Sick leave or retired due to pain & $11(31.4)$ & $5(15.2)$ & \\
\hline Retired unrelated to pain & $21(60)$ & $23(69.6)$ & \\
\hline Work satisfaction $-/ 100^{\mathrm{a}}$ & $83.8+7.5$ & $86.2 \pm 10.9$ & 0.49 \\
\hline \multicolumn{4}{|l|}{ Operated vertebral segment(s) } \\
\hline 1 level & $18(53 \%)$ & $16(53 \%)$ & 0.97 \\
\hline 2 levels & $8(24 \%)$ & $8(27 \%)$ & 0.77 \\
\hline 3 levels & $7(20 \%)$ & $6(20 \%)$ & 0.95 \\
\hline 4 levels & $1(3 \%)$ & $0(0 \%)$ & - \\
\hline \multicolumn{4}{|l|}{ Primary outcomes } \\
\hline Leg pain intensity $-/ 10$ & $7.4 \pm 1.8$ & $6.7 \pm 2.3$ & 0.16 \\
\hline \multicolumn{4}{|l|}{ Secondary outcomes } \\
\hline \multicolumn{4}{|l|}{ Clinical outcomes } \\
\hline Back pain intensity-/10 & $5.3 \pm 3.0$ & $5.5 \pm 2.7$ & 0.77 \\
\hline Leg pain dominant-n (\%) & $27(77)$ & $22(66)$ & 0.33 \\
\hline Weekly days with pain-(/7) & $6.9 \pm 0.1$ & $6.9 \pm 0.3$ & 0.62 \\
\hline Back disability-/100 & $37.2 \pm 16.4$ & $37.4 \pm 11.9$ & 0.93 \\
\hline Kinesiophobia-/68 & $47.4 \pm 8.0$ & $45.2 \pm 7.7$ & 0.24 \\
\hline Depression-/63 & $4.4 \pm 5.0$ & $4.7 \pm 4.1$ & 0.79 \\
\hline \multicolumn{4}{|l|}{ Physical outcomes } \\
\hline \multicolumn{4}{|l|}{ Lumbar active ROMs-degrees } \\
\hline Flexion & $66.0 \pm 25.1$ & $67.2 \pm 20.8$ & 0.84 \\
\hline Extension & $14.4 \pm 7.0$ & $14.4 \pm 5.4$ & 0.99 \\
\hline Left lateral flexion & $13.0 \pm 7.3$ & $12.0 \pm 6.1$ & 0.58 \\
\hline Right lateral flexion & $13.8 \pm 7.9$ & $13.7 \pm 6.8$ & 0.97 \\
\hline \multicolumn{4}{|l|}{ Trunk muscles strength $-\mathrm{N}$ m } \\
\hline Flexion & $46.2 \pm 23.4$ & $44.1 \pm 28.1$ & 0.66 \\
\hline Extension & $33.2 \pm 28.8$ & $28.4 \pm 24.2$ & 0.47 \\
\hline Right knee extensor strength-lbs & $62.1 \pm 31.7$ & $60.7 \pm 28.6$ & 0.84 \\
\hline Left knee extensor strength—lbs & $72.6 \pm 27.3$ & $75.6 \pm 26.9$ & 0.77 \\
\hline Lumbar extensor endurance-s & $45.2 \pm 59.9$ & $38.7 \pm 49.2$ & 0.64 \\
\hline \multicolumn{4}{|l|}{ Walking capacities-s } \\
\hline Time to 1st symptoms & $116.8 \pm 87.4$ & $97.4 \pm 93.4$ & 0.38 \\
\hline Total ambulation time & $196.7 \pm 94.0$ & $190.0 \pm 139.1$ & 0.81 \\
\hline \multicolumn{4}{|l|}{ Newly added outcomes ${ }^{b}$} \\
\hline LSS disability—(/55) & $39.5 \pm 4.2$ & $38.0 \pm 4.2$ & 0.40 \\
\hline Sit to stand-repetitions & $9.6 \pm 3.8$ & $9.0 \pm 2.7$ & 0.65 \\
\hline Get up and go-s & $8.3 \pm 1.5$ & $9.8 \pm 3.8$ & 0.23 \\
\hline
\end{tabular}

Table 1. Participants' baseline characteristics. EMG electromyography, ODI Oswestry disability index, LSS

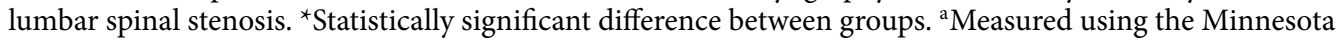
Satisfaction Questionnaire ${ }^{37}$, results based on 4 participants from the intervention group and 5 from the control group (those employed or on sick leave at the time of the study). ${ }^{b}$ For the newly added outcomes, 15 and 13 participants provided data from the intervention and control group respectively. 


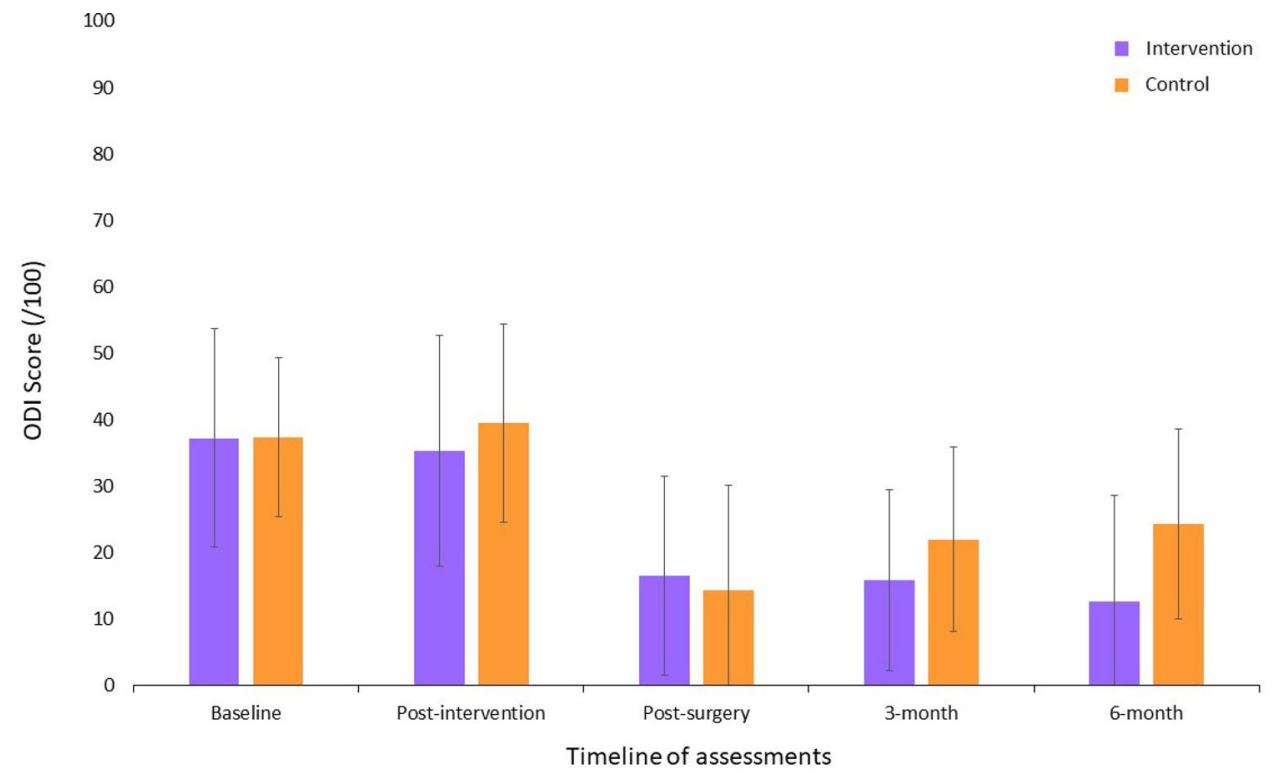

Figure 2. Comparison of low back related disability (means \pm SD) (Greater scores indicate greater disability; ODI Oswestry disability index).

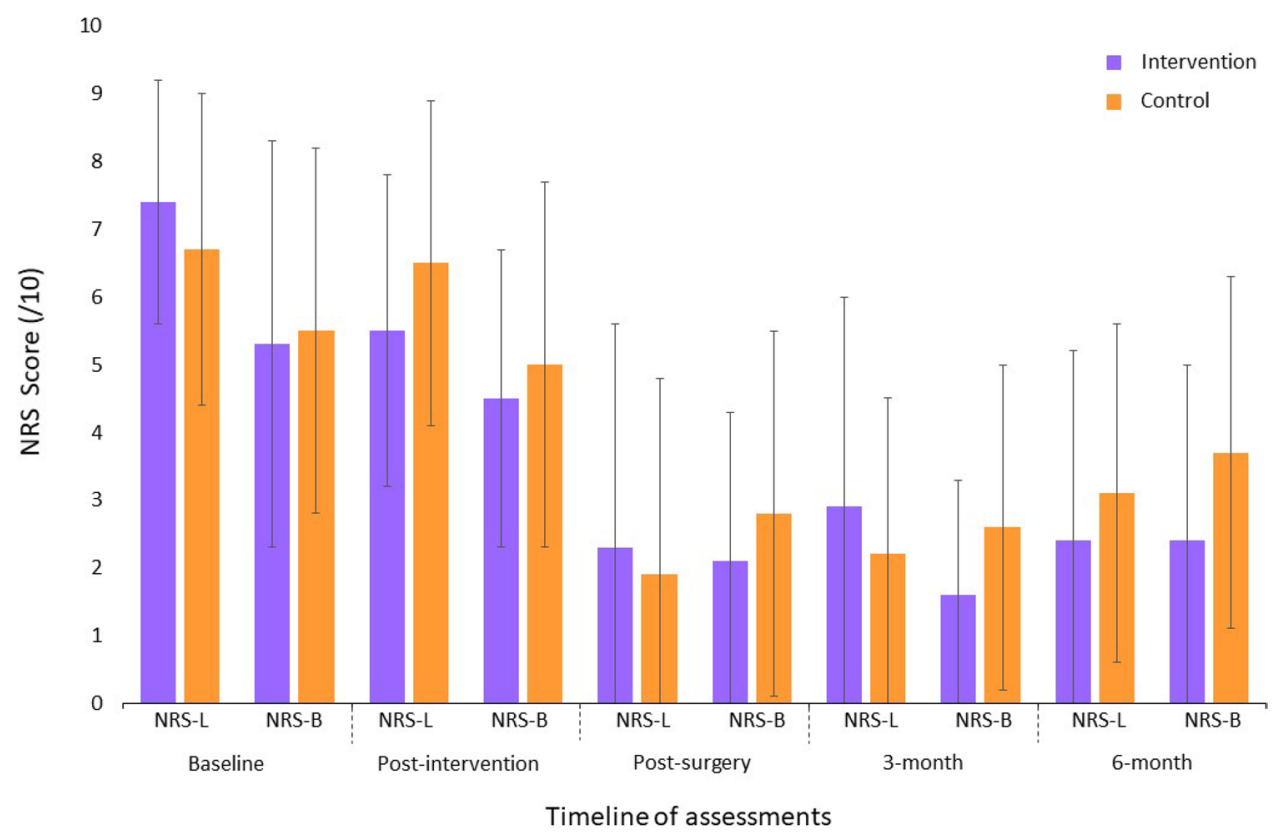

Figure 3. Comparison of leg and back pain intensity (means \pm SD) (Greater scores indicate greater pain intensity; $N R S-L$ numerical rating scale for leg pain intensity, $N R S-B$ numerical rating scale for low back pain intensity).

fidence intervals for each group are presented based on clinical and physical outcomes for all time points in Tables 2 and 3 respectively.

Quality of life. Changes in quality of life were assessed by comparing the proportions of individuals from each group that reported an improvement in each of the questionnaire 5 dimensions. No between group significant difference was found at any of the assessment timepoints (all ps $>0.05$ ).

Participants' perceived change in global status. At the preoperative assessment, participants in the intervention group reported greater positive change in their global status (mean \pm SD: $2.9 \pm 1.3$ ) compared to the control group $(4.5 \pm 1.0)$. Sixty-nine per cents reported that their status had "improved" (=very much better, much better, or slightly better) in the intervention group compared to $11.5 \%$ in the control group $(\mathrm{p}<0.001)$. 


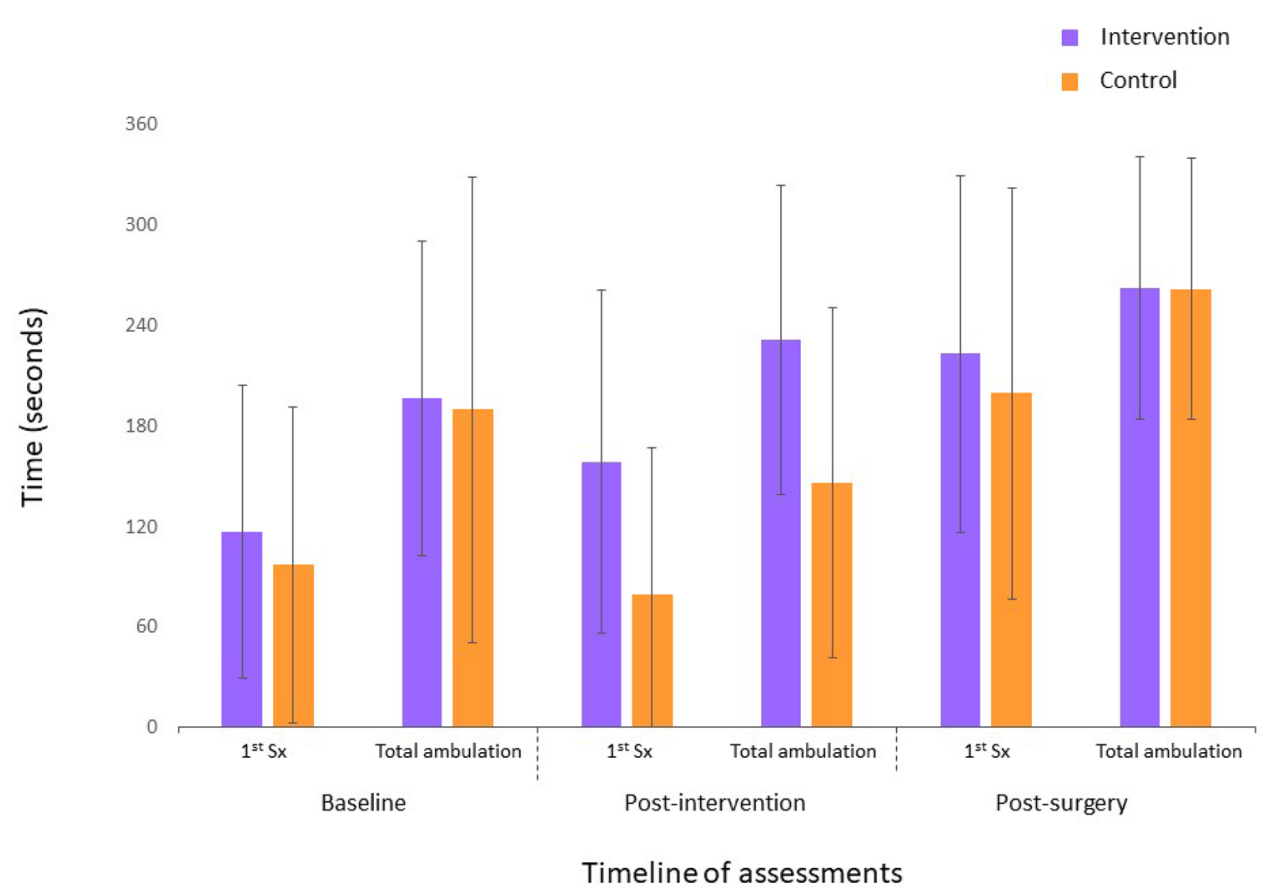

Figure 4. Comparison of walking capacities (means \pm SD) (Greater scores indicate better walking capacities; $1 s t$ Sx walking time to first symptoms).

Similarly, $13 \%$ percent reported that their status had "worsened" (=very much worse, much worse, or slightly worse) in the intervention group compared with $46 \%$ in the control group $(\mathrm{p}<0.01)$.

Participants' satisfaction. Thirty-one participants provided an objective evaluation of their overall satisfaction with the intervention program. The mean satisfaction score (mean $\pm \mathrm{SD})$ reached $94.4 \% \pm 8.3$. Both groups provided similar satisfaction rates with regards to postoperative back pain outcome with a score (mean \pm SD) of $84.4 \% \pm 22.2$ for the intervention group and of $84.0 \% \pm 22.5$ for the control group $(p=0.23)$. Satisfaction rates for postoperative leg pain outcome were also similar with $82.3 \% \pm 23.4$ in the intervention group and $84.6 \% \pm 23.9$ in the control group $(\mathrm{p}=0.34)$.

Intraoperative data. Intraoperative measures and length of hospital stay were similar in both groups. Results are presented in Table 4.

Harms. At no point in time were adverse events reported as a result of either the training program or physical assessments.

\section{Discussion}

The aim of the present study was to assess the effectiveness of an exercise-based prehabilitation program, compared to usual care, on improving preoperative capacities, and postoperative recovery in patients with lumbar spinal stenosis. The results showed improvements in both self-reported clinical and objective physical outcomes at the post-intervention assessment in favor of the prehabilitation group. However, theses differences were not maintained after the surgery. As such, in the postoperative phase, back-related disability was the only parameter that followed distinct trajectories between groups, with improvements seen in the intervention group and deteriorations seen in the control group, over the 6-month follow-up.

Clinical significance. The within group differences observed in the intervention group after the prehabilitation intervention were clinically significant for decreased leg pain intensity ${ }^{38}$ ( -1.9 point), LSS-related disability $^{38}$ ( -4.5 points), and the sit-to-stand test ${ }^{39}(+2.4$ repetitions) (major clinically important improvement determined from patients with hip osteoarthritis $\geq 2$ ). To the best of our knowledge no minimal clinically important difference has been determined for maximum isometric flexor strength and trunk extensor endurance. We noted a $13.7 \%$ increase in trunk flexor strength $(+6.4 \mathrm{Nm})$ and a $45 \%$ improvement in low back extensor endurance (+20.5 s) from baseline. On the other hand, improvement in total ambulation time $(+34.9 \mathrm{~s}=17.7 \%)$ did not reach the proposed $30 \%$ threshold for clinical significance ${ }^{40}$.

The between group differences identified for low back extensor endurance ( $+47.8 \mathrm{~s}=267.0 \%$ increase) and total ambulation time $(+85.4 \mathrm{~s}=58.4 \%$ increase $)$ correspond to a large and medium effect size, respectively ${ }^{41}$. 


\begin{tabular}{|c|c|c|c|c|c|c|c|}
\hline \multirow[b]{2}{*}{ Variable } & \multirow[b]{2}{*}{$\mathbf{N}$} & \multirow{2}{*}{\begin{tabular}{|l|} 
Intervention group \\
Mean + SD (95\% \\
CI)
\end{tabular}} & \multirow{2}{*}{$\mathrm{N}$} & \multirow{2}{*}{$\begin{array}{l}\text { Control group } \\
\text { Mean + SD (95\% } \\
\text { CI) }\end{array}$} & \multirow[b]{2}{*}{$\begin{array}{l}\text { Main effect of } \\
\text { time }(p)\end{array}$} & \multirow[b]{2}{*}{$\begin{array}{l}\text { Main effect of } \\
\text { group }(p)\end{array}$} & \multirow[b]{2}{*}{$\begin{array}{l}\text { Group } \times \text { time } \\
\text { interaction }(p)\end{array}$} \\
\hline & & & & & & & \\
\hline \multicolumn{8}{|c|}{ Back pain intensity/10 } \\
\hline Baseline & 35 & $5.4 \pm 3.0(4.3$ to 6.4$)$ & 33 & $5.6 \pm 2.7(4.6$ to 6.5$)$ & \multirow{2}{*}{0.09} & \multirow{2}{*}{0.69} & \multirow{2}{*}{0.91} \\
\hline Post-intervention & 29 & $4.5 \pm 2.2(3.6$ to 5.3$)$ & 26 & $5.0 \pm 2.7(3.9$ to 6.1$)$ & & & \\
\hline Post-surgery & 32 & $2.1 \pm 2.2(1.3$ to 2.9$)$ & 21 & $2.8 \pm 2.7(1.5$ to 3.9$)$ & \multirow{3}{*}{0.01} & \multirow{3}{*}{0.17} & \multirow{3}{*}{0.90} \\
\hline 3 months & 24 & $1.6 \pm 1.7(0.9$ to 2.3$)$ & 23 & $2.6 \pm 2.4(1.5$ to 3.6$)$ & & & \\
\hline 6 months & 24 & $2.4 \pm 2.6(1.3$ to 3.5$)$ & 19 & $3.7 \pm 2.6(2.4$ to 4.9$)$ & & & \\
\hline \multicolumn{8}{|c|}{ Leg pain intensity/10 } \\
\hline Baseline & 35 & $7.4 \pm 1.9(6.8$ to 8.1$)$ & 33 & $6.7 \pm 2.4(5.9$ to 7.6$)$ & \multirow{2}{*}{0.001} & \multirow{2}{*}{0.76} & \multirow{2}{*}{0.03} \\
\hline Post-intervention & 29 & $5.5 \pm 2.3(4.6$ to 6.4$)$ & 26 & $6.5 \pm 2.4(5.5$ to 7.4$)$ & & & \\
\hline Post-surgery & 32 & $2.3 \pm 3.3(1.1$ to 3.5$)$ & 21 & $1.9 \pm 2.9(0.6$ to 3.2$)$ & \multirow{3}{*}{0.11} & \multirow{3}{*}{0.99} & \multirow{3}{*}{0.30} \\
\hline 3 months & 24 & $2.9 \pm 3.1(1.6$ to 4.3$)$ & 23 & $2.2 \pm 2.3(1.2$ to 3.2$)$ & & & \\
\hline 6 months & 24 & $2.4 \pm 2.8(1.2$ to 3.6$)$ & 19 & $3.1 \pm 2.5(1.8$ to 4.3$)$ & & & \\
\hline \multicolumn{8}{|c|}{ LSS disability/55 or $79^{*}$} \\
\hline Baseline & 15 & $\begin{array}{l}39.5 \pm 4.3 \text { (36.6 to } \\
42.4)\end{array}$ & 13 & $\begin{array}{l}38.0 \pm 4.2(35.5 \text { to } \\
40.6)\end{array}$ & \multirow{2}{*}{0.005} & \multirow{2}{*}{0.47} & \multirow{2}{*}{0.04} \\
\hline Post-intervention & 9 & $\begin{array}{l}35.0 \pm 7.9(28.9 \text { to } \\
41.0)\end{array}$ & 8 & $\begin{array}{l}37.5 \pm 6.5 \text { (32.1 to } \\
42.9)\end{array}$ & & & \\
\hline Post-surgery ${ }^{\star}$ & 13 & $\begin{array}{l}29.6 \pm 8.5(24.5 \text { to } \\
34.7)\end{array}$ & 6 & $\begin{array}{l}28.5 \pm 10.6(17.4 \\
\text { to } 39.6)\end{array}$ & & & \\
\hline 3 months ${ }^{*}$ & 8 & $\begin{array}{l}37.9 \pm 10.2(29.3 \text { to } \\
46.4)\end{array}$ & 6 & $\begin{array}{l}39.2 \pm 14.4(24.0 \\
\text { to } 54.3)\end{array}$ & 0.27 & 0.72 & 0.83 \\
\hline 6 months $^{*}$ & 9 & $\begin{array}{l}30.5 \pm 10.1(22.7 \text { to } \\
38.4)\end{array}$ & 3 & $\begin{array}{l}45.0 \pm 21.6(8.8 \text { to } \\
78.8)\end{array}$ & & & \\
\hline Back disability/10 & & & & & & & \\
\hline Baseline & 35 & $\begin{array}{l}37.2 \pm 16.5(32.5 \text { to } \\
42.9)\end{array}$ & 33 & $\begin{array}{l}37.5 \pm 11.9(33.2 \\
\text { to } 41.7)\end{array}$ & 0.95 & 0.29 & 0.12 \\
\hline Post-intervention & 29 & $\begin{array}{l}35.3 \pm 17.4(28.7 \text { to } \\
41.9)\end{array}$ & 26 & $\begin{array}{l}39.5 \pm 14.9(33.5 \\
\text { to } 45.5)\end{array}$ & 0.95 & 0.29 & 0.12 \\
\hline Post-surgery & 32 & $\begin{array}{l}16.5 \pm 15.0(11.0 \text { to } \\
22.0)\end{array}$ & 21 & $\begin{array}{l}14.3 \pm 15.8 \text { (7.1 to } \\
21.5)\end{array}$ & & & \\
\hline 3 months & 24 & $\begin{array}{l}15.8 \pm 13.6(10.1 \text { to } \\
21.6)\end{array}$ & 23 & $\begin{array}{l}22.0 \pm 13.9(15.9 \\
\text { to } 28.0)\end{array}$ & 0.02 & 0.36 & 0.003 \\
\hline 6 months & 24 & $\begin{array}{l}12.7 \pm 16.0(6.1 \text { to } \\
19.4)\end{array}$ & 19 & $\begin{array}{l}24.3 \pm 14.3(17.6 \\
\text { to } 31.0)\end{array}$ & & & \\
\hline Kinesiophobia/68 & & & & & & & \\
\hline Baseline & 35 & $\begin{array}{l}47.5 \pm 8.1(44.7 \text { to } \\
50.3)\end{array}$ & 33 & $\begin{array}{l}45.2 \pm 7.7(42.5 \text { to } \\
47.9)\end{array}$ & 0.74 & 0.90 & $0.02^{\ddagger}$ \\
\hline Post-intervention & 29 & $\begin{array}{l}44.2 \pm 7.7(41.3 \text { to } \\
47.1)\end{array}$ & 26 & $\begin{array}{l}47.6 \pm 8.0(44.4 \text { to } \\
50.8)\end{array}$ & & & \\
\hline Post-surgery & 32 & $\begin{array}{l}38.2 \pm 8.9(35.0 \text { to } \\
41.4)\end{array}$ & 21 & $\begin{array}{l}38.9 \pm 9.3(34.7 \text { to } \\
43.2)\end{array}$ & & & \\
\hline 3 months & 24 & $\begin{array}{l}37.9 \pm 7.8(34.7 \text { to } \\
41.1)\end{array}$ & 23 & $\begin{array}{l}41.3 \pm 7.3(38.1 \text { to } \\
44.5)\end{array}$ & 0.47 & 0.48 & 0.78 \\
\hline 6 months & 24 & $\begin{array}{l}37.6 \pm 7.6(34.5 \text { to } \\
40.7)\end{array}$ & 19 & $\begin{array}{l}40.4 \pm 10.7(35.5 \\
\text { to } 45.3)\end{array}$ & & & \\
\hline Depression/63 & & & & & & & \\
\hline Baseline & 35 & $4.4 \pm 5.1(2.7$ to 6.2$)$ & 33 & $4.7 \pm 4.2(3.2$ to 6.2$)$ & 0.42 & 0.28 & 0.97 \\
\hline Post-intervention & 29 & $4.3 \pm 5.3(2.3$ to 6.3$)$ & 26 & $5.3 \pm 5.2(3.2$ to 7.3$)$ & 0.42 & 0.20 & 0.71 \\
\hline Post-surgery & 32 & $2.0 \pm 3.4(0.8$ to 3.3$)$ & 21 & $1.8 \pm 3.1(0.4$ to 3.3$)$ & & & \\
\hline 3 months & 24 & $2.7 \pm 3.5(1.3$ to 4.2$)$ & 23 & $3.9 \pm 4.6(1.9$ to 5.9$)$ & 0.009 & 0.87 & 0.87 \\
\hline 6 months & 24 & $1.7 \pm 2.0(0.8$ to 2.5$)$ & 19 & $3.4 \pm 3.3(1.9$ to 4.9$)$ & & & \\
\hline
\end{tabular}

Table 2. Results for clinical outcome measures. SD standard deviation, CI confidence interval. ${ }^{\star}$ LSSrelated disability measured using the Swiss Spinal Stenosis Questionnaire-maximum score at baseline and post-intervention assessments is 55 (includes symptoms and function subscales) and maximum score at postoperative, 3-month and 6-month assessments is 79 (includes symptoms, function and satisfaction subscales). ${ }^{\ddagger}$ The Group $\times$ Time interaction term was statistically significant but post-hoc analysis using Bonferroni test revealed no significant within or between group differences. Bold indicate a significant intragroup change from baseline (for analyses conducted between baseline and post-intervention assessments) or intra-group change from post-surgery (for analyses conducted between post-surgery and follow-ups assessments). 


\begin{tabular}{|c|c|c|c|c|c|c|c|}
\hline \multirow[b]{2}{*}{ Variable } & \multirow[b]{2}{*}{$\mathbf{N}$} & \multirow{2}{*}{\begin{tabular}{|l|} 
Intervention group \\
Mean + SD (95\% CI) \\
\end{tabular}} & \multirow[b]{2}{*}{$\mathbf{N}$} & \multirow{2}{*}{\begin{tabular}{|l|} 
Control group \\
Mean +SD (95\% CI)
\end{tabular}} & \multirow[b]{2}{*}{ Main effect of time $(p)$} & \multirow[b]{2}{*}{ Main effect of group $(p)$} & \multirow{2}{*}{$\begin{array}{l}\text { Group } \times \text { time interaction } \\
(p)\end{array}$} \\
\hline & & & & & & & \\
\hline \multicolumn{8}{|c|}{ Trunk muscles strength $\mathbf{N} \mathbf{m}$} \\
\hline \multicolumn{8}{|c|}{ Flexion } \\
\hline Baseline & 35 & $46.8 \pm 23.4(38.7$ to 54.9$)$ & 33 & $44.1 \pm 28.1(33.7$ to 54.3$)$ & \multirow{2}{*}{0.02} & \multirow{2}{*}{0.16} & \multirow{2}{*}{0.03} \\
\hline Post-intervention & 29 & $53.2 \pm 24.8(43.4$ to 63.1$)$ & 26 & $42.1 \pm 26.1$ (30.5 to 53.7$)$ & & & \\
\hline Post-surgery & 32 & $54.4 \pm 28.5$ (43.1 to 65.7$)$ & 21 & $50.1 \pm 27.8(37.9$ to 63.9$)$ & \multicolumn{3}{|l|}{0.32} \\
\hline \multicolumn{8}{|l|}{ Extension } \\
\hline Baseline & 35 & $32.8 \pm 29.2(22.8$ to 42.9$)$ & 33 & $26.7 \pm 26.3$ (17.2 to 36.2$)$ & \multirow{2}{*}{0.004} & \multirow{2}{*}{0.21} & \multirow{2}{*}{0.51} \\
\hline Post-intervention & 29 & $57.0 \pm 61.0(32.4$ to 81.6$)$ & 26 & $26.3 \pm 26.7$ (14.1 to 38.5$)$ & & & \\
\hline Post-surgery & 32 & $46.4 \pm 41.3(29.8$ to 63.1$)$ & 21 & $48.5 \pm 40.0(29.2$ to 67.8$)$ & 0.85 & & \\
\hline \multicolumn{8}{|c|}{ Lumbar active ROMs (degrees) } \\
\hline \multicolumn{8}{|c|}{ Flexion } \\
\hline Baseline & 35 & $66.0 \pm 25.2$ (56.9 to 75.1$)$ & 33 & $67.2 \pm 20.9(59.1$ to 75.3$)$ & ( & O & (1) \\
\hline Post-intervention & 29 & $68.6 \pm 27.2(57.6$ to 79.6$)$ & 26 & $62.3 \pm 25.8$ (50.6 to 74.0$)$ & 0.69 & 0.12 & 0.15 \\
\hline Post-surgery & 32 & $68.8 \pm 17.6$ (61.9 to 75.8$)$ & 21 & $72.9 \pm 19.4(63.6$ to 82.3$)$ & 0.45 & & \\
\hline Extension & & & & & & & \\
\hline Baseline & 35 & $14.4 \pm 7.1$ (11.7 to 17.2$)$ & 33 & $14.4 \pm 5.5(12.0$ to 16.8$)$ & 028 & م10 & 005 \\
\hline Post-intervention & 29 & $16.8 \pm 6.9(13.9$ to 19.7$)$ & 26 & $12.7 \pm 7.1(9.4$ to 15.9$)$ & 0.28 & 0.10 & 0.05 \\
\hline Post-surgery & 32 & $17.7 \pm 6.7(15.1$ to 20.4$)$ & 21 & $15.2 \pm 5.9(12.4$ to 18.0$)$ & 0.15 & & \\
\hline Left lateral flexion & & & & & & & \\
\hline Baseline & 35 & $13.0 \pm 7.3(10.4$ to 15.7$)$ & 33 & $12.1 \pm 6.2(9.7$ to 14.5$)$ & 0.97 & 030 & 037 \\
\hline Post-intervention & 29 & $14.1 \pm 8.8(10.6$ to 17.6$)$ & 26 & $10.6 \pm 5.3(8.2$ to 13.0$)$ & 0.97 & & \\
\hline Post-surgery & 32 & $13.5 \pm 5.7(11.3$ to 15.8$)$ & 21 & $12.8 \pm 5.4(10.2$ to 15.4$)$ & 0.63 & & \\
\hline Right lateral flexion & & & & & & & \\
\hline Baseline & 35 & $13.8 \pm 7.9(10.9$ to 16.7$)$ & 33 & $13.8 \pm 6.8(11.1$ to 16.4$)$ & 026 & 065 & 020 \\
\hline Post-intervention & 29 & $13.2 \pm 8.2(9.9$ to 16.4$)$ & 26 & $11.2 \pm 5.5(8.7$ to 13.7$)$ & 0.26 & 0.65 & 0.29 \\
\hline Post-surgery & 32 & $14.4 \pm 6.6(11.8$ to 17.1$)$ & 21 & $15.3 \pm 7.9(11.5$ to 19.1$)$ & 0.75 & & \\
\hline Right knee extenso & stre & ngth-lbs & & & & & \\
\hline Baseline & 35 & $62.2 \pm 31.7$ (51.3 to 73.1$)$ & 33 & $60.5 \pm 28.7$ (50.6 to 70.9$)$ & O & O & 054 \\
\hline Post-intervention & 29 & $65.6 \pm 29.9(53.5$ to 77.7$)$ & 26 & $54.2 \pm 31.5(41.2$ to 67.2$)$ & 0.45 & 0.20 & 0.54 \\
\hline Post-surgery & 32 & $75.7 \pm 37.6(61.7$ to 89.8$)$ & 21 & $62.4 \pm 28.3$ ( 49.5 to 75.3$)$ & 0.49 & & \\
\hline Left knee extensor & tren & th-lbs & & & & & \\
\hline Baseline & 35 & $72.6 \pm 27.3$ (55.3 to 89.9$)$ & 33 & $75.6 \pm 26.9(60.1$ to 91.2$)$ & O & 054 & 020 \\
\hline Post-intervention & 29 & $75.4 \pm 26.2(55.3$ to 95.5$)$ & 26 & $69.3 \pm 31.9(46.5$ to 92.1$)$ & 0.45 & 0.34 & 0.20 \\
\hline Post-surgery & 32 & $87.5 \pm 24.4$ (72.8 to 102.3$)$ & 21 & $80.7 \pm 27.4$ (55.3 to 106.0$)$ & 0.30 & & \\
\hline Lumbar extensors & dur: & ince-s & & & & & \\
\hline Baseline & 32 & $45.2 \pm 59.9$ (23.6 to 66.8$)$ & 30 & $38.8 \pm 49.2(20.4$ to 57.1$)$ & (n) & 014 & 0002 \\
\hline Post-intervention & 27 & $65.7 \pm 65.6(39.8 \text { to } 91.7)^{\star}$ & 23 & $17.9 \pm 35.8(2.4 \text { to } 33.4)^{\star}$ & 0.61 & 0.14 & 0.002 \\
\hline Post-surgery & 30 & $69.5 \pm 59.2$ (47.4 to 91.6$)$ & 21 & $57.3 \pm 60.5(29.8$ to 84.8$)$ & 0.88 & & \\
\hline Walking capacities & & & & & & & \\
\hline Time to 1st sympto & & & & & & & \\
\hline Baseline & 35 & $116.8 \pm 87.4(86.8$ to 146.8$)$ & 33 & $97.4 \pm 93.4$ (63.1 to 131.7 ) & م & 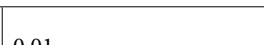 & noe \\
\hline Post-intervention & 29 & $158.8 \pm 102.4(119.8$ to 197.7$)$ & 25 & $79.4 \pm 87.4$ (43.3 to 115.4$)$ & 0.44 & 0.01 & 0.08 \\
\hline Post-surgery & 30 & $223.1 \pm 106.5$ (182.6 to 263.6$)$ & 21 & $199.6 \pm 122.8(143.7$ to 255.5$)$ & 0.39 & & \\
\hline Total ambulation ti & & & & & & & \\
\hline Baseline & 35 & $196.8 \pm 94.0(163.9$ to 229.6$)$ & 33 & $190.1 \pm 139.1$ (139.9 to 240.2$)$ & & & \\
\hline Post-intervention & 29 & $231.7 \pm 92.2(195.9 \text { to } 267.5)^{*}$ & 25 & $\begin{array}{l}146.3 \pm 104.7(103.1 \text { to } \\
189.5)^{*}\end{array}$ & 0.81 & 0.03 & 0.02 \\
\hline Post-surgery & 30 & $262.2 \pm 78.5(232.3$ to 292.0$)$ & 21 & $262.0 \pm 78.1$ (226.5 to 297.6$)$ & 0.85 & & \\
\hline Continued & & & & & & & \\
\hline
\end{tabular}




\begin{tabular}{|c|c|c|c|c|c|c|c|}
\hline \multirow[b]{2}{*}{ Variable } & \multirow[b]{2}{*}{$\mathbf{N}$} & \multirow{2}{*}{$\begin{array}{l}\text { Intervention group } \\
\text { Mean + SD (95\% CI) }\end{array}$} & \multirow[b]{2}{*}{$\mathbf{N}$} & \multirow{2}{*}{\begin{tabular}{|l|} 
Control group \\
Mean + SD (95\% CI) \\
\end{tabular}} & \multirow[b]{2}{*}{ Main effect of time ( $p$ ) } & \multirow[b]{2}{*}{ Main effect of group $(p)$} & \multirow{2}{*}{$\begin{array}{l}\text { Group } \times \text { time interaction } \\
(p)\end{array}$} \\
\hline & & & & & & & \\
\hline \multicolumn{8}{|l|}{ Get up and go-s } \\
\hline Baseline & 15 & $8.8 \pm 1.7$ (7.7 to 9.9$)$ & 13 & $10.2 \pm 4.1(7.7$ to 12.7$)$ & \multirow{2}{*}{0.007} & \multirow{2}{*}{0.15} & \multirow{2}{*}{0.12} \\
\hline Post-intervention & 9 & $7.5 \pm 1.9(6.0$ to 8.9$)$ & 8 & $9.7 \pm 2.6$ (7.7 to 11.7$)$ & & & \\
\hline Post-surgery & 13 & $6.7 \pm 1.3(5.9$ to 7.5$)$ & 6 & $8.0 \pm 2.7(5.2$ to 10.9$)$ & 0.09 & & \\
\hline \multicolumn{8}{|c|}{ Sit to stand-repetitions } \\
\hline Baseline & 15 & $9.7 \pm 3.8(7.2$ to 12.1$)$ & 13 & $9.1 \pm 2.7$ (7.4 to 10.7$)$ & \multirow{2}{*}{0.001} & \multirow{2}{*}{0.14} & \multirow{2}{*}{0.01} \\
\hline Post-intervention & 9 & $12.1 \pm 3.6(9.4$ to 14.9$)$ & 8 & $8.4 \pm 2.2(6.8$ to 10.1$)$ & & & \\
\hline Post-surgery & 13 & $11.8 \pm 2.2(10.4$ to 13.1$)$ & 6 & $12.0 \pm 2.1$ (9.8 to 14.2$)$ & \multicolumn{3}{|l|}{0.89} \\
\hline
\end{tabular}

Table 3. Results for physical outcome measures. $S D$ standard deviation, $C I$ confidence interval, $R O M$ ranges of motion. Bold indicate a significant intra-group change from baseline. ${ }^{\star}$ Denotes a significant between group difference.

\begin{tabular}{|l|l|l|l|}
\hline & $\begin{array}{l}\text { Intervention }(\mathbf{n}=\mathbf{3 4}) \\
\text { Mean } \pm \text { SD }\end{array}$ & $\begin{array}{l}\text { Control }(\mathbf{n}=30) \\
\text { Mean } \pm \text { SD }\end{array}$ & $p$ \\
\hline Length of surgery (min) & $101.3 \pm 47.9$ & $105.2 \pm 57.8$ & 0.77 \\
\hline Blood loss (ml) & $213.4 \pm 243.1$ & $221.0 \pm 226.3$ & 0.90 \\
\hline Intraoperative complication (n) & 0 & 2 & - \\
\hline Length of hospital stay (days) & $4.1 \pm 3.2$ & $4.5 \pm 2.0$ & 0.58 \\
\hline Minimally invasive surgey (n) & 11 & 8 & 0.62 \\
\hline Open surgery (n) & 23 & 22 & 0.27 \\
\hline Received physiotherapy postoperatively (n) & 3 & 3 & 0.87 \\
\hline
\end{tabular}

Table 4. Perioperative data. Physiotherapy consisted of one hospital-based or home-based visit to ensure adequate independency. The intraoperative complications were dural tears.

Comparison with other trials. As of today, only few publications have investigated the effectiveness of prehabilitation interventions within the context of spine surgery. In the systematic review published in early 2021 by Janssen et al. a total of 15 studies were included, most of which (13/15) investigated cognitive behavioral therapy. The authors concluded based on meta-analyses that there was very low to moderate quality evidence that prehabilitation has no effect compared to usual care on physical functioning, leg and back pain intensity, health-related quality of life, depression, anxiety, length of hospital stay, and analgesics use. The present trial adds on to the review of Janssen et al. by being the first to report on adverse event related to both the prehabilitation intervention and physical assessments. In addition, we did not exclude patients with multiple comorbidities or with previous history of surgery and imposed no maximum age limit, which were identified as limitations in previous studies.

Results of the present study somewhat contrast with the results previously reported by Nielsen et al. ${ }^{16}$ which included greater function prior to surgery, and faster postoperative recovery and discharge from hospital in favor of the intervention group compared to the standard care group. Their proposed intervention combined 6 to 8 weeks of preoperative daily individualized home training program, preoperative supplemental food intake, and early in-hospital postoperative rehabilitation. In comparison, we decided to include only one aspect of the recommended prehabilitation triad (exercise training, nutrition, and emotional wellbeing) ${ }^{6}$ in order to tease out the effects of exercises alone given that evidence on how to best prepare for spine surgery is scarce. However, there is evidence from studies investigating major surgeries that supports the use of multimodal interventions, including modification of behavioural and lifestyle risk factors, to improve surgical outcome rather than solely focusing on the underlying disease process ${ }^{42}$. In addition, despite the fact that the focus of prehabilitation has primarily been put on the optimisation of physical comorbidities, there is an increasing body of evidence that emphasizes the role of preoperative psychological factors ${ }^{43}$ on both physical and psychological postoperative outcomes. Likewise, patients with high-risks profiles, such as frailty and comorbidity, have been proposed to be the ones that would most benefit from prehabilitation ${ }^{44-46}$. Thus, pre-operative risk stratification taking into account both modifiable and non-modifiable risk factors of poor surgical outcome and complications would allow to tailor prehabilitation interventions to the patients' needs and capacities ${ }^{42,47}$. Nevertheless, such endeavour to optimize patients' preoperative status requires multidisciplinary input and substantial resources to ensure proper monitoring.

Strengths and limitations. Considering the limited available evidence on the effect of prehabilitation interventions within the context of spine surgery, the results of the present study should be interpreted with caution. Among its strengths, the study followed a randomised, controlled design and complied with the related Consolidated Standards of Reporting Trials guideline. Furthermore, the proposed intervention could be suited 
to individual participants' level of physical capacities and no substantial protocol modifications were required to accommodate day-to-day variation in patients' symptomatology. The intervention was also delivered by a single certified kinesiologist to decrease the probability of a clinician effect and avoid inter-clinician variations. Of importance, the study reflects a pragmatic approach to rehabilitation intervention within the Quebec public health care system, characterized by the variable intervention length based on the surgical waitlist. Participating individuals came from both Trois-Rivières and its surrounding areas, which allows to infer the results to other Canadian provinces with comparable public health care system. However, the results cannot be extrapolated to patients with spinal instability or primary foraminal stenosis. Based on the collaborating neurosurgeons' experience, patients requiring fusion surgery (instrumented or not) or foraminotomy would undergo more complex procedures and have different recovery pathways and were therefore not included in the study. With regards to the methods, missing data were dealt with using multiple imputations so that reasonable power could be maintained when conducting the statistical analyses.

In contrast, the study also has limitations, of which the first one is the use of the pilot study data. Although using data from pilot study to conduct sample size estimate is widely debated in the literature, there was no pooled results available at the time the study was conducted ${ }^{48}$. In addition, estimating variance from a small pool of data may inflate the type I error rate when conducting the main study ${ }^{49}$. Also, it will not be possible in future meta-analysis to use the data from both the pilot study and the main trial because the data of pilot study have been included in the main study and are no longer independent.

Similarly, the fact that the sample size fell short of the targeted number at enrollment combined with a high drop out rate increased data heterogeneity which limits the power for some of the analyses. On the one hand, recruitment was hampered by self-perceived health-related barriers and transportation issues. To have provided education at the time of recruitment with regards to the benefits of being active, beyond study purposes, could have improved participation rate ${ }^{47}$. Likewise, to have offered the option to perform the exercise program at home, in the instance where it could be done safely without supervision, could have facilitated recruitment ${ }^{50}$. On the other hand, the decision to prematurely stopped recruitment due to unforeseen organizational and time constraints at the local hospital which significantly limited the referral pathway from the neurosurgery unit also played a role in having a small final sample size.

With regards to limitations in the conduct of the study, the principal investigator was not blind to participants' group allocation while conducting the assessments, which may have led to measurement bias. Finally, considering that adding outcomes after the pilot phase of the study resulted in fewer than half of the participants providing data (Swiss Spinal Stenosis questionnaire, get up and go test, and sit to stand test) which greatly limit their interpretation, these results should be viewed as preliminary.

Overall, the current body of evidence on the effectiveness of prehabilitation program designed for spine surgery is less robust than that of other surgical contexts and appears to be less promising. Considering that patients awaiting elective surgery may have longer preoperative windows and, for some, lesser complex clinical profiles than those awaiting non-elective surgery, preoperative interventions may play a different role than augmenting fitness for surgery. As such, many of the participants in the present study did not seek conservative care prior to undergoing surgery. Given that for patients with stable clinical status, a trial of conservative care is recommended prior to surgical management ${ }^{51}$, preoperative interventions may be beneficial in terms of clinical improvements and allow to better detect those for whom surgery is necessary to regain satisfactory functional capacities.

\section{Conclusion}

The main objective of the present study was to evaluate the effectiveness of a 6-week preoperative exercise-based program, compared to usual care, in patients awaiting elective surgery for LSS. Our findings suggest that while the intervention yielded improvements on clinical status and physical capacities preoperatively, it was insufficient to foster a more rapid shot-term postoperative recovery. Tailored prehabilitation based on stratification of highrisk patient profiles coupled with education should make the object of future studies looking at preoperative intervention in the context of spinal surgery.

Received: 11 December 2020; Accepted: 12 May 2021

Published online: 26 May 2021

\section{References}

1. Carli, F., Bessissow, A., Awasthi, R. \& Liberman, S. Prehabilitation: Finally utilizing frailty screening data. Eur. J. Surg. Oncol. 46, 321-325. https://doi.org/10.1016/j.ejso.2020.01.001 (2020).

2. Schricker, T. et al. Parenteral nutrition and protein sparing after surgery: Do we need glucose?. Metab. Clin. Exp. 56, 1044-1050. https://doi.org/10.1016/j.metabol.2007.03.013 (2007).

3. Kehlet, H. \& Wilmore, D. W. Evidence-based surgical care and the evolution of fast-track surgery. Ann. Surg. 248, 189-198. https:// doi.org/10.1097/SLA.0b013e31817f2cla (2008).

4. Ljungqvist, O., Scott, M. \& Fearon, K. C. Enhanced recovery after surgery: A review. JAMA Surg. 152, 292-298. https://doi.org/ 10.1001/jamasurg.2016.4952 (2017).

5. Hawkins, S. A. \& Wiswell, R. A. Rate and mechanism of maximal oxygen consumption decline with aging. Sports Med. 33, 877-888. https://doi.org/10.2165/00007256-200333120-00002 (2003).

6. Carli, F. \& Ferreira, V. Prehabilitation: A new area of integration between geriatricians, anesthesiologists, and exercise therapists. Aging Clin. Exp. Res. 30, 241-244. https://doi.org/10.1007/s40520-017-0875-8 (2018).

7. Carli, F. \& Zavorsky, G. S. Optimizing functional exercise capacity in the elderly surgical population. Curr. Opin. Clin. Nutr. Metab. Care 8, 23-32. https://doi.org/10.1097/00075197-200501000-00005 (2005).

8. Topp, R., Ditmyer, M., King, K., Doherty, K. \& Hornyak, J. 3rd. The effect of bed rest and potential of prehabilitation on patients in the intensive care unit. AACN Clin. Issues 13, 263-276 (2002).

9. Carli, F. \& Scheede-Bergdahl, C. Prehabilitation to enhance perioperative care. Anesthesiol. Clin. 33, 17-33. https://doi.org/10. 1016/j.anclin.2014.11.002 (2015). 
10. Puts, M. T. E. et al. Interventions to prevent or reduce the level of frailty in community-dwelling older adults: A scoping review of the literature and international policies. Age Ageing 46, 383-392. https://doi.org/10.1093/ageing/afw247 (2017).

11. Carli, F. \& Ferreira, V. Prehabilitation: A new area of integration between geriatricians, anesthesiologists, and exercise therapists. Aging Clin. Exp. Res. https://doi.org/10.1007/s40520-017-0875-8 (2018).

12. Treanor, C., Kyaw, T. \& Donnelly, M. An international review and meta-analysis of prehabilitation compared to usual care for cancer patients. J. Cancer Surviv. Res. Pract. 12, 64-73. https://doi.org/10.1007/s11764-017-0645-9 (2018).

13. Santa Mina, D. et al. Effect of total-body prehabilitation on postoperative outcomes: A systematic review and meta-analysis. Physiotherapy 100, 196-207. https://doi.org/10.1016/j.physio.2013.08.008 (2014).

14. Gometz, A. et al. The effectiveness of prehabilitation (prehab) in both functional and economic outcomes following spinal surgery: A systematic review. Cureus 10, e2675. https://doi.org/10.7759/cureus.2675 (2018).

15. Janssen, E. R. C. et al. Current prehabilitation programs do not improve the postoperative outcomes of patients scheduled for lumbar spine surgery: A systematic review with meta-analysis. J. Orthop. Sports Phys. Ther. 51, 103-114. https://doi.org/10.2519/ jospt.2021.9748 (2021)

16. Nielsen, P. R., Jorgensen, L. D., Dahl, B., Pedersen, T. \& Tonnesen, H. Prehabilitation and early rehabilitation after spinal surgery: Randomized clinical trial. Clin. Rehabil. 24, 137-148. https://doi.org/10.1177/0269215509347432 (2010).

17. Sobottke, R. et al. Predictors of surgical, general and follow-up complications in lumbar spinal stenosis relative to patient age as emerged from the Spine Tango Registry. Eur. Spine J. 21, 411-417. https://doi.org/10.1007/s00586-011-2016-y (2012).

18. Deyo, R. A. Treatment of lumbar spinal stenosis: A balancing act. Spine J. 10, 625-627. https://doi.org/10.1016/j.spinee.2010.05. $006(2010)$

19. Fanuele, J. C., Birkmeyer, N. J., Abdu, W. A., Tosteson, T. D. \& Weinstein, J. N. The impact of spinal problems on the health status of patients: Have we underestimated the effect?. Spine 25, 1509-1514 (2000).

20. Norden, J., Smuck, M., Sinha, A., Hu, R. \& Tomkins-Lane, C. Objective measurement of free-living physical activity (performance) in lumbar spinal stenosis: Are physical activity guidelines being met?. Spine J. 17, 26-33. https://doi.org/10.1016/j.spinee.2016.10. 016 (2017).

21. Weinstein, J. N. et al. Surgical compared with nonoperative treatment for lumbar degenerative spondylolisthesis: Four-year results in the spine patient outcomes research trial (SPORT) randomized and observational cohorts. J. Bone Jt. Surg. Am. 91, 1295-1304. https://doi.org/10.2106/JBJS.H.00913 (2009).

22. Marchand, A. A. et al. Effects of a prehabilitation program on patients' recovery following spinal stenosis surgery: Study protocol for a randomized controlled trial. Trials 16, 483. https://doi.org/10.1186/s13063-015-1009-2 (2015).

23. Marchand, A. A. et al. Feasibility of conducting an active exercise prehabilitation program in patients awaiting spinal stenosis surgery: A randomized pilot study. Sci. Rep. 9, 12257. https://doi.org/10.1038/s41598-019-48736-7 (2019).

24. Hawker, G. A., Mian, S., Kendzerska, T. \& French, M. Measures of adult pain: Visual analog scale for pain (VAS Pain), numeric rating scale for pain (NRS Pain), McGill Pain questionnaire (MPQ), short-form McGill pain questionnaire (SF-MPQ), chronic pain grade scale (CPGS), short form-36 bodily pain scale (SF-36 BPS), and measure of intermittent and constant osteoarthritis pain (ICOAP). Arthritis Care Res. 63(Suppl 11), S240-252. https://doi.org/10.1002/acr.20543 (2011).

25. Vogler, D., Paillex, R., Norberg, M., de Goumoens, P. \& Cabri, J. Cross-cultural validation of the Oswestry disability index in French. Ann. Readapt. et de Med. Phys. 51, 379-385. https://doi.org/10.1016/j.annrmp.2008.03.006 (2008).

26. Coons, S. J., Rao, S., Keininger, D. L. \& Hays, R. D. A comparative review of generic quality-of-life instruments. Pharmacoeconomics 17, 13-35 (2000).

27. French, D. J., Roach, P. J. \& Mayes, S. Peur du mouvement chez des accidentés du travail: L'Échelle de Kinésiophobie de Tampa (EKT). Can. J. Behav. Sci. / Revue Can. des Sci. du Comport. 34, 28-33 (2002).

28. Bourque, P. \& Beaudette, D. Étude psychometrique du questionnaire de dépression de Beck auprès d'un échantillon détudiants universitaires francophones (Psychometric study of the Beck Depression Inventory on a sample of French-speaking university students). Can. J. Behav. Sci./Revue Can. des Sci. du Comport. 14, 211-218 (1982).

29. Dworkin, R. H. et al. Core outcome measures for chronic pain clinical trials: IMMPACT recommendations. Pain 113, 9-19. https:// doi.org/10.1016/j.pain.2004.09.012 (2005).

30. Champagne, A., Descarreaux, M. \& Lafond, D. Comparison between elderly and young males' lumbopelvic extensor muscle endurance assessed during a clinical isometric back extension test. J. Manip. Physiol. Ther. 32, 521-526. https://doi.org/10.1016/j. jmpt.2009.08.008 (2009).

31. Juan-Recio, C., López-Plaza, D., Barbado Murillo, D., García-Vaquero, M. P. \& Vera-García, F. J. Reliability assessment and correlation analysis of 3 protocols to measure trunk muscle strength and endurance. J. Sports Sci. 36, 357-364. https://doi.org/10. 1080/02640414.2017.1307439 (2018).

32. Padulo, J. et al. Validity and reliability of isometric-bench for knee isometric assessment. Int. J. Environ. Res. Public Health 17, 4326. https://doi.org/10.3390/ijerph17124326 (2020).

33. MacDermid, J. C., Arumugam, V., Vincent, J. I., Payne, K. L. \& So, A. K. Reliability of three landmarking methods for dual inclinometry measurements of lumbar flexion and extension. BMC Musculoskelet. Disord. 16, 121. https://doi.org/10.1186/s12891015-0578-2 (2015).

34. Jones, C. J., Rikli, R. E. \& Beam, W. C. A 30-s chair-stand test as a measure of lower body strength in community-residing older adults. Res. Q. Exerc. Sport 70, 113-119. https://doi.org/10.1080/02701367.1999.10608028 (1999).

35. Podsiadlo, D. \& Richardson, S. The timed "up \& go": A test of basic functional mobility for frail elderly persons. J. Am. Geriatr. Soc. 39, 142-148. https://doi.org/10.1111/j.1532-5415.1991.tb01616.x (1991).

36. Marchand, A. A., Tetreau, C., O'Shaughnessy, J. \& Descarreaux, M. French-Canadian adaptation and validation of the Swiss spinal stenosis questionnaire for patients with lumbar spinal stenosis. Spine 44, E487-e493. https://doi.org/10.1097/brs.0000000000002896 (2019).

37. Roussel, P. P. Rémunération, Motivation et Satisfaction au Travail (Economica, Collection Recherche en Gestion, 1996).

38. Cleland, J. A., Whitman, J. M., Houser, J. L., Wainner, R. S. \& Childs, J. D. Psychometric properties of selected tests in patients with lumbar spinal stenosis. Spine J. 12, 921-931. https://doi.org/10.1016/j.spinee.2012.05.004 (2012).

39. Wright, A. A., Cook, C. E., Baxter, G. D., Dockerty, J. D. \& Abbott, J. H. A Comparison of 3 methodological approaches to defining major clinically important improvement of 4 performance measures in patients with hip osteoarthritis. J. Orthop. Sports Phys. Ther. 41, 319-327. https://doi.org/10.2519/jospt.2011.3515 (2011).

40. Schneider, M. J. et al. Comparative clinical effectiveness of nonsurgical treatment methods in patients with lumbar spinal stenosis: A randomized clinical trial. JAMA Netw. Open 2, e186828. https://doi.org/10.1001/jamanetworkopen.2018.6828 (2019).

41. Richardson, J. T. E. Eta squared and partial eta squared as measures of effect size in educational research. Educ. Res. Rev. 6, 135-147. https://doi.org/10.1016/j.edurev.2010.12.001 (2011).

42. Durrand, J., Singh, S. J. \& Danjoux, G. Prehabilitation. Clin. Med. (Lond.) 19, 458-464. https://doi.org/10.7861/clinmed.2019-0257 (2019).

43. Levett, D. Z. H. \& Grimmett, C. Psychological factors, prehabilitation and surgical outcomes: Evidence and future directions. Anaesthesia 74(Suppl 1), 36-42. https://doi.org/10.1111/anae.14507 (2019).

44. Carli, F. \& Baldini, G. From preoperative assessment to preoperative optimization of frail older patiens. Eur. J. Surg. Oncol. 47, 519-523. https://doi.org/10.1016/j.ejso.2020.06.011 (2021). 
45. Hulzebos, E. H. \& van Meeteren, N. L. Making the elderly fit for surgery. Br. J. Surg. 103, e12-e15. https://doi.org/10.1002/bjs. 10033 (2016).

46. Glance, L. G., Osler, T. M. \& Neuman, M. D. Redesigning surgical decision making for high-risk patients. N. Engl. J. Med. 370, 1379-1381. https://doi.org/10.1056/NEJMp1315538 (2014).

47. Levett, D. Z., Edwards, M., Grocott, M. \& Mythen, M. Preparing the patient for surgery to improve outcomes. Best Pract. Res. Clin. Anaesthesiol. 30, 145-157. https://doi.org/10.1016/j.bpa.2016.04.002 (2016).

48. Thabane, L. et al. A tutorial on pilot studies: The what, why and how. BMC Med. Res. Methodol. 10, 1. https://doi.org/10.1186/ 1471-2288-10-1 (2010).

49. Coffey, C. S. \& Muller, K. E. Properties of internal pilots with the univariate approach to repeated measures. Stat. Med. 22, 2469-2485. https://doi.org/10.1002/sim.1466 (2003).

50. Ferreira, V. et al. Maximizing patient adherence to prehabilitation: What do the patients say?. Support Care Cancer 26, 2717-2723. https://doi.org/10.1007/s00520-018-4109-1 (2018).

51. Siebert, E. et al. Lumbar spinal stenosis: Syndrome, diagnostics and treatment. Nat. Rev. Neurol. 5, 392-403. https://doi.org/10. 1038/nrneurol.2009.90 (2009).

\section{Acknowledgements}

The authors would like to acknowledge Mr. Pierre-Luc Yao for the assistance with the statistical analyses and thank the neurosurgery team from the CIUSSS-MCQ and the patients who generously participated in the study.

\section{Author contributions}

A.A.M. contributed to the conception and design of the study, data acquisition, analysis and interpretation and drafting of the manuscript. M.H. contributed to the design of the study and data acquisition. J.O., C.E.C. and V.C. contributed to the design of the study and revision of the manuscript. M.D. contributed to the conception and design of the study, data analysis and interpretation and drafting of the manuscript. All authors reviewed and approved the final version of the manuscript.

\section{Funding}

This study was funded by the Chaire de recherche internationale en santé neuromusculosquelettique and its partners the Centre intégré universitaire de santé et de services sociaux de la Mauricie-et-du-Centre-du-Québec (CIUSSSMCQ) and the Université du Québec à Trois-Rivières. AAM received a doctoral scholarship by the Institut de recherche Robert-Sauvé en santé et en sécurité du travail (IRSST) and the Fonds de recherche du Québec-Santé (FRQS).

\section{Competing interests}

The authors declare no competing interests.

\section{Additional information}

Correspondence and requests for materials should be addressed to A.-A.M.

Reprints and permissions information is available at www.nature.com/reprints.

Publisher's note Springer Nature remains neutral with regard to jurisdictional claims in published maps and institutional affiliations.

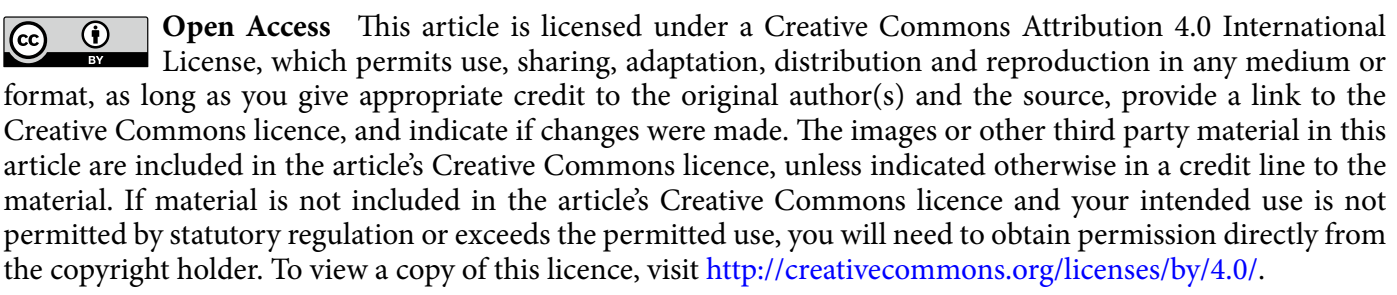

(C) The Author(s) 2021 